\title{
Mathematical Journal of Interdisciplinary Sciences
}

\section{Scientific Numerical Pattern in Stringed-Fretted Musical Instrument}

\author{
Anindita Roy Chowdhury and Naresh Sharma* \\ School of Basic and Applied Sciences, GD Goenka University, Gurugram-122103, Haryana, India \\ "Email: naresh.sharma2006@gmail.com
}

\section{ARTICLE INFORMATION}

Received: December 18, 2019

Revised: January 15, 2020

Accepted: January 24, 2020

Published Online: March 30, 2020

Keywords:

Correlation, Fretted, Harmonics, Stringed and Trendline

\author{
ABSTRACT
}

Music, a creative art has a strong foundation on science and mathematics. Source of music can vary from vocal chord to various types of musical instruments. One of the popular stringed and fretted musical instrument, guitar has been discussed here. The structure of the guitar is based on mathematical and scientific concepts. Harmonics and frequency play pivotal role in generation of music from a guitar. In this paper, the authors have investigated various factors related to the structure of a guitar. Aspects related to the musical notes of a guitar have been analyzed to gain a better insight into the mathematical pattern involved in the music of a guitar.

\section{Introduction}

A proper sequence of simple sounds conforming to certain rules that are governed by the logic of Mathematics and the laws of Physics creates music. Pitch, duration and loudness are the three basic elements of music. According to scientific perspective, pitch is related to frequency; similarly, duration is related to time and loudness is connected to energy. Melodious music can be generated from the human vocal chord or various types of instruments that usually belong to different categories of musical instruments like stringed, percussion, piped (Blatter 2016; Bhattacharya 1989).

Stringed instruments can be further sub-divided into three types-plucked, bowed, struck. Among the popular instruments, guitar, and the Indian sitar belong to the plucked category, violin and the Indian sarangi are from the bowed section, and western piano and Indian santoor belong to the struck category.

Plucking gives an impulse that causes the string to vibrate in different modes of vibration and creates harmonics that results in standing waves. With the onset of vibrations in a string, there are certain points in it where the amplitude of the string vibration is zero from mean position; technically, such points are called nodes and midway between two consecutive nodes, lies the antinode where the string vibrates with maximum amplitude (Bhattacharya 1989).
When a string vibrates with maximum amplitude at the centre of its length, fundamental mode or first harmonic results. With increase in number of nodes in between, harmonics increases (Blatter 2016; Gunther 2012). The relationship between length $(L)$ of the string and wavelength $(\lambda)$ of sound corresponding to $\mathrm{n}^{\text {th }}$ harmonic can be written as $L=n(\lambda / 2)$. Length is connected to frequency $(f)$ and velocity $(v)$ of the sound generated through the relation, $f=v / 2 L$. If $f_{1}=$ fundamental frequency, then frequency of higher order harmonics are given by the relation, $f_{n}=n f_{1}$. A string of finite length in a guitar will generate music when plucked. Standing waves in a stringed instrument occur at certain specific frequencies that can be predicted by Mersenne's Law (Gunther 2012; "Determining Pitch" 2017).

The other factors on which frequency depends are length and mass per unit length. Mercenne's law illustrates that the frequency of the vibrating string is inversely proportional to the length of the string. Accordingly, the $\mathrm{n}$-th harmonic of the string occurs at frequency, $f_{n}=(n / 2 L) \sqrt{T / \mu}$ where $n=$ harmonic number $=1,2,3, \ldots ; \mathrm{L}=$ length of vibrating string, $\mu=$ mass per unit length of the vibrating string.

On plucking a guitar string, it vibrates exhibiting a variety of sounds. When the string of a guitar is plucked at different sites along its length, there is a significant variance 
in a note's sound. It has been noticed that notes plucked near the bridge of the guitar seem to have a brighter, sharper sound whereas the tone that comes from it being plucked over the sound hole is different. Physically, the speed of these waves depends on the tension (stiffness) in the string and its mass density or mass per unit length. The guitar with the stiffer spring will vibrate at a higher frequency than the system with the looser spring that in turn would affect frequency (Herman 2012; Gualandri 2016) Wave velocity along the string is given by the expression, $v=\sqrt{T / \mu}$. On changing the length of the vibrating string, the vibration will occur at different frequencies. As the player changes his finger's position from one fret to the other, then the vibrating length of the strings vary. The six guitar strings have different mass per unit length. The ones with higher mass per unit length will vibrate with lower frequency than the thinner strings (Varieschi and Gower 2010).

In this paper, the authors aim to investigate multiple numerical aspects associated with the structure and hence, the music obtained from a stringed and fretted musical instrument. Here, the experimental instrument chosen is guitar. The mathematical characteristics of the string diameters, fret heights, inter-fret distance and length of musical notes were investigated through this work.

\section{Methodology}

To study different characteristics of a stringed and fretted instrument, guitar was chosen; both acoustic and electric. Irrespective of the guitar type, it has six strings. In standard classical guitars, usually there are 20 frets whereas in electric guitars the number of frets vary from 21 to 24 .

\subsection{Measurement of String Diameter}

With a screw gauge of least count $0.01 \mathrm{~mm}$, diameters of the six strings were measured. By convention, the thinnest string is considered as the first string, denoted as $\mathrm{S} 1$ and the thickest as S6, here (Savage and Andrew 2017). To obtain a good average, string diameter was measured at the neck and body of the guitar and also at the mid of the fret board. The values and their respective analysis are shown in the Results Section (Table 1-2 and Figure 1). For the acoustic guitar, string diameter has been plotted as a function of its mass per unit length (Figure 2).

\subsection{Measurement of Fret Height and Inter-fret Distance}

Digital caliper having a least count of $0.01 \mathrm{~mm}$ was used to measure the heights of the fret and also the inter-fret distance, i.e. distance between two consecutive frets. In order to understand the structure of a fret, the measurement was taken at the left and right edges of the fret; it was carried out even at the centre of the fret. (Table 3 and Figure 3). Due to the crucial placement of the frets, digital caliper was a preferred tool in comparison to other standard tools of least count $0.01 \mathrm{~mm}$. The distance between two consecutive frets has been designated as inter-fret distance. The values have been plotted for both acoustic and electric guitars in Figure 4.

\subsection{Measurement of Note Length}

The twelve musical notes, A, A\#, B, C, C\#, D, D\#, E, F, F\#, G, G\# were played on each of the six strings in an Acoustic Guitar. The respective lengths were noted with a standard scale of least count $1 \mathrm{~mm}$ on which statistical analysis was done for further revelation.

\section{Results and Discussion}

In Table 1, the diameter measurement of the Acoustic Guitar is shown.

Table 1: Diameter of the Strings of Acoustic Guitar.

\begin{tabular}{|l|l|l|l|l|}
\hline \multirow{2}{*}{$\begin{array}{l}\text { String } \\
\text { No. }\end{array}$} & \multicolumn{2}{|c|}{$\begin{array}{l}\text { Diameter of Acoustic Guitar String at } \\
\text { Different Positions }\end{array}$} & $\begin{array}{l}\text { Mean } \\
\text { Diameter } \\
\text { (cm) }\end{array}$ \\
\cline { 2 - 5 } & $\begin{array}{l}\text { Neck of } \\
\text { Guitar }\end{array}$ & $\begin{array}{l}\text { Mid of } \\
\text { Fretboard }\end{array}$ & $\begin{array}{l}\text { Body of } \\
\text { Guitar }\end{array}$ & \\
\hline 1 & 0.22 & 0.22 & 0.22 & 0.22 \\
2 & 0.26 & 0.26 & 0.26 & 0.26 \\
3 & 0.37 & 0.37 & 0.37 & 0.37 \\
4 & 0.62 & 0.62 & 0.62 & 0.62 \\
5 & 0.84 & 0.84 & 0.84 & 0.84 \\
6 & 1.05 & 1.05 & 1.05 & 1.05 \\
\hline
\end{tabular}

Thus, it is evident from Table 1 that the diameter of each string is uniform throughout but different from the other strings. In Table 2, the diameters of the Electric Guitar are noted.

Table 2: Diameter of the Strings of Electric Guitar.

\begin{tabular}{|l|l|l|l|l|}
\hline \multirow{2}{*}{$\begin{array}{l}\text { String } \\
\text { No. }\end{array}$} & \multicolumn{2}{|c|}{$\begin{array}{l}\text { Diameter of Acoustic Guitar String at } \\
\text { Different Positions }\end{array}$} & $\begin{array}{l}\text { Mean } \\
\text { Diameter } \\
\text { Diam }\end{array}$ \\
\cline { 2 - 4 } & $\begin{array}{l}\text { Neck of } \\
\text { Guitar }\end{array}$ & $\begin{array}{l}\text { Mid of } \\
\text { Fretboard }\end{array}$ & $\begin{array}{l}\text { Body of } \\
\text { Guitar }\end{array}$ & \\
\hline 1 & 0.32 & 0.32 & 0.32 & 0.32 \\
2 & 0.40 & 0.40 & 0.40 & 0.40 \\
3 & 0.62 & 0.62 & 0.62 & 0.62 \\
4 & 0.84 & 0.84 & 0.84 & 0.84 \\
5 & 1.06 & 1.06 & 1.06 & 1.06 \\
6 & 1.34 & 1.34 & 1.34 & 1.34 \\
\hline
\end{tabular}


Here, too, it is noted that the diameter of each string is uniform throughout and the diameter of each string is unique. At the same time, both Table 1 and Table 2 shows that the diameter of every string is not only different from each other but they are placed from one end to the other along the length of the guitar in an increasing trend of the diameter. So, further investigation was carried out to find out a relation among this variation of string diameter.

Plot in Figure 1 indicates the exponential variation of the diameter of the guitar strings. In Figure 2, the nature of variation of string diameter in an Acoustic Guitar is analyzed with reference to the standard value of mass per unit length of the string (Noyce 2017). Two trendlines, linear and polynomial have been plotted in Figure 2.

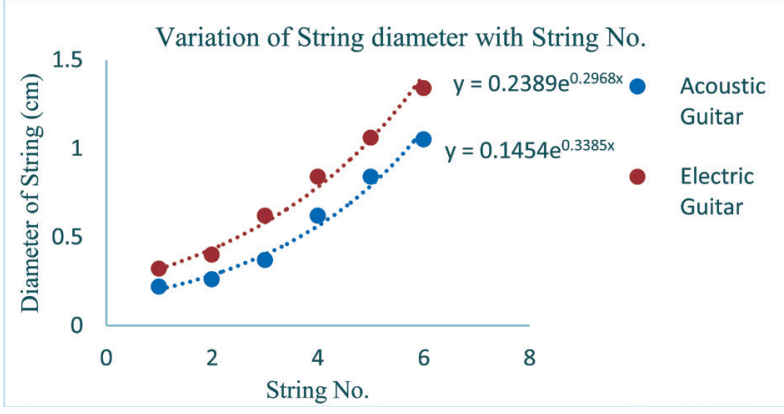

Figure 1: Variation in Diameter of Strings in Acoustic and Electric Guitars

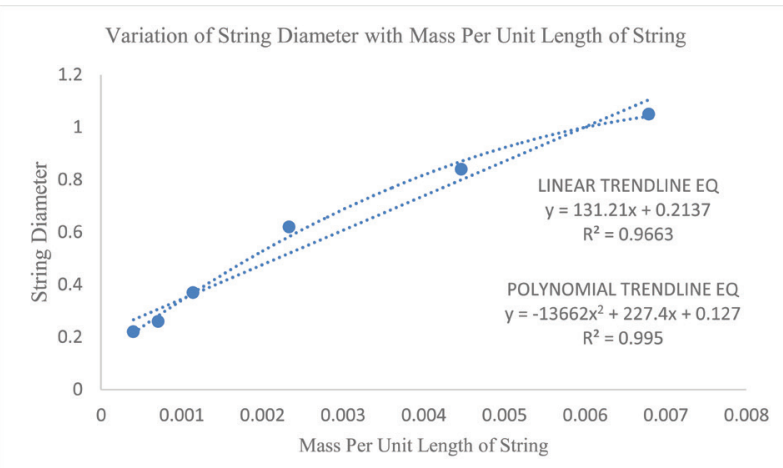

Figure 2: Variation of String Diameter of Acoustic Guitar with Standard Mass Per Unit Length of String

Figure 2 supports the fact that the diameter of the strings in a guitar variesexponentially. It has been noticed that the mass density and tension of guitar strings are determinant factors for velocity of the wave along the string. Speed of the wave along a string under high tension would be high and consequently, frequency would be higher for a given wavelength. For pleasant music, waves must travel with varied speed along the different strings and so either the tension or the mass density of the strings should vary. Usually, the tension along the strings are nearly the same but the thickness of the string varies resulting in varied mass density, and hence, generation of pleasant music. The string with larger mass density experiences lower speed of the waves along it (Hokin 2018). The variation in the string diameters facilitate an approximately similar tension on each string with the maintenance of different speeds along the various strings in order to facilitate the musician.

\subsection{Measurement of Fret Height}

The heights of every fret in both acoustic and electric guitars were measured. Values are presented in Table 3. It can be observed that the height of the frets are always highest at the central level and it progressively decreases from fret no. 1 to fret no. 20; fret no. 1 has been considered, here towards the neck of the guitar.

Table 3: Fret Height in Acoustic Guitar and Electric Guitar

\begin{tabular}{|c|c|c|c|c|c|c|c|}
\hline \multirow{2}{*}{$\begin{array}{l}\text { Fret } \\
\text { No. }\end{array}$} & \multicolumn{3}{|c|}{ Acoustic Guitar } & \multirow{2}{*}{$\begin{array}{l}\text { Fret } \\
\text { No. }\end{array}$} & \multicolumn{3}{|c|}{ Electric Guitar } \\
\hline & Right & Central & Left & & Right & Central & Left \\
\hline 1 & 1.11 & 1.15 & 1.12 & 1 & 1.04 & 1.05 & 0.95 \\
\hline 2 & 1.06 & 1.09 & 1.04 & 2 & 0.98 & 1.08 & 0.93 \\
\hline 3 & 0.9 & 0.96 & 0.95 & 3 & 1.01 & 1.06 & 0.92 \\
\hline 4 & 0.92 & 0.95 & 0.93 & 4 & 1 & 1.03 & 0.9 \\
\hline 5 & 0.93 & 0.96 & 0.9 & 5 & 0.92 & 0.97 & 0.85 \\
\hline 6 & 0.85 & 0.97 & 0.84 & 6 & 0.93 & 0.99 & 0.89 \\
\hline 7 & 0.95 & 0.97 & 0.9 & 7 & 0.84 & 0.87 & 0.86 \\
\hline 8 & 0.94 & 0.99 & 0.96 & 8 & 1.09 & 1.16 & 1.02 \\
\hline 9 & 0.94 & 0.97 & 0.85 & 9 & 0.96 & 1.01 & 0.77 \\
\hline 10 & 0.93 & 0.99 & 0.97 & 10 & 0.96 & 1.02 & 0.89 \\
\hline 11 & 0.96 & 0.98 & 0.96 & 11 & 1 & 1,02 & 0.82 \\
\hline 12 & 0.96 & 0.98 & 0.92 & 12 & 1.02 & 1.03 & 0.97 \\
\hline 13 & 0.93 & 0.94 & 0.85 & 13 & 0.99 & 1.01 & 0.98 \\
\hline 14 & 0.98 & 0.99 & 0.93 & 14 & 1.02 & 1.03 & 0.9 \\
\hline 15 & 0.93 & 1.09 & 0.93 & 15 & 1.03 & 1.05 & 0,97 \\
\hline 16 & 0.98 & 1.09 & 0.94 & 16 & 1.04 & 1.09 & 0.92 \\
\hline 17 & 0.95 & 1.04 & 0.95 & 17 & 0.97 & 1.05 & 0.9 \\
\hline 18 & 0.94 & 1.01 & 0.96 & 18 & 0.84 & 0.87 & 0.83 \\
\hline 19 & 0.99 & 1.03 & 0.88 & 19 & 0.88 & 0.99 & 0.83 \\
\hline 20 & 0.93 & 0.95 & 0.92 & 20 & 0.92 & 1.03 & 0.87 \\
\hline \multirow{2}{*}{\multicolumn{4}{|c|}{$\begin{array}{l}{ }^{*} \text { Value of Fret Height }(\mathrm{mm}) \\
{ }^{*} \text { Measurement Position for Fret } \\
\text { Height }\end{array}$}} & 21 & 1.03 & 1.05 & 0.99 \\
\hline & & & & 22 & 0.97 & 1.03 & 0.87 \\
\hline
\end{tabular}


The above variation is plotted in Figure 3 wherein only the central height of the frets have been chosen.

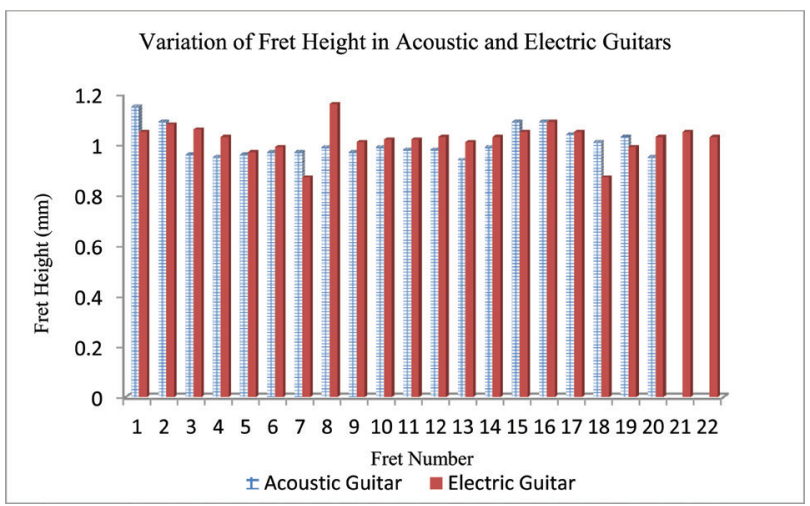

Figure 3: Variation of Fret Height in Acoustic Guitar and Electric Guitar.

From Figure 3, it can be noted that in case of majority of the frets, the central height of the fret is slightly higher in case of the electric guitar in comparison to the acoustic guitar. On pressing a string against the different frets, the vibrating length of that string reduces to the distance between the bridge of the guitar and finger on the specific fret, thus, producing different musical notes. The height of the frets are not much to enable easier string bending to produce clear notes without excess pressure (Drozdowski 2012).

Variation in the inter-fret distance, i.e. the distance between the consecutive frets was also plotted in Figure 4. From the plot, it is evident that the space between two consecutive frets is in the (exponentially) decreasing order. The frets are placed along the fingerboard of a guitar according to the fret rule that enunciates the placement of frets at one-eighteenth the remaining length of the string. This indicates that the frets are placed approximately a semitone apart. Usually, a musical octave is divided into 12 equal tempered semitones. In this paper, the authors have illustrated graphically the precise manner in which the interfret distance have changed in Figure 4.

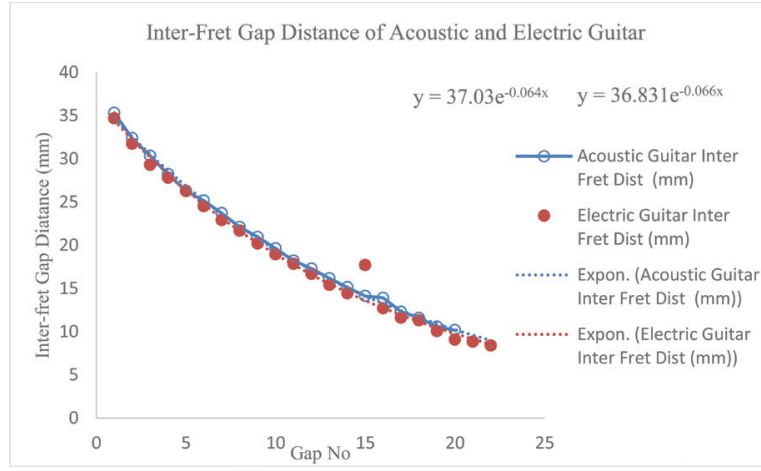

Figure 4: Variation of Inter-fret Distance in Acoustic Guitar and Electric Guitar.
The exponential relation that exists for the inter-fret distance of the acoustic guitar examined is $y=37.03 \mathrm{e}^{-0.064 x}$ and for the electric guitar is $y=36.831 \mathrm{e}^{-0.066 x}$. Existing work shows that a fret is placed at a distance of $1 / 1.0595$ from the bridge, the second fret at a distance of $1 / 1.0595^{2}$ and so on (Hokin 2018). This indicates the existence of a specific pattern or a mathematical ratio according to which the distance between consecutive frets change. Here, the authors could show the exponential relation on the basis of which the frets have been placed along the finger board in a guitar. The frets are located appropriately to match the exact length for a specific note along every string. During this work, the length of the different notes along every string was measured and a cyclical pattern was noted among the various note lengths. In Table 4, the correlation coefficient between the strings based on the length of the notes are shown (Nave 2017; McNeil 2017)

Table 4 : Correlation Coefficient Matrix Between Strings.

\begin{tabular}{|l|l|l|l|l|l|l|}
\hline String & S1 & S2 & S3 & S4 & S5 & S6 \\
\hline S1 & - & -0.45 & -0.13 & 0.27 & -0.56 & 1.00 \\
S2 & -0.45 & - & -0.33 & -0.13 & 0.14 & -0.45 \\
S3 & -0.13 & -0.33 & - & -0.45 & 0.14 & -0.13 \\
S4 & 0.14 & -0.13 & -0.45 & - & -0.45 & 0.14 \\
S5 & -0.45 & 0.14 & 0.14 & -0.45 & - & -0.45 \\
S6 & 1.00 & -0.45 & -0.13 & 0.14 & -0.45 & - \\
\hline
\end{tabular}

In Table 4, the result of correlation between the same strings have not been considered as their strong correlation is obvious. From the values in Table 4, it can be inferred that string S1 and string $S 6$ are strongly correlated. With tension and mass density as constant, the length for the various notes of the octave is known to be a function of the original string length (Hokin 2018). A statistical analysis was carried out on the data of the lengths of the notes along the six different guitar strings of an acoustic guitar. The results are tabulated in Table 5.

Table 5: Descriptive Statistics of Length of Musical Notes in Acoustic Guitar.

\begin{tabular}{|l|l|l|l|l|l|l|}
\hline Statistical & \multicolumn{6}{|c|}{ String No. } \\
\cline { 2 - 7 } Parameters & $\mathbf{S 1}$ & $\mathbf{S 2}$ & $\mathbf{S 3}$ & $\mathbf{S 4}$ & $\mathbf{S 5}$ & $\mathbf{S 6}$ \\
\hline Mean & 25.16 & 25.16 & 25.16 & 25.16 & 25.16 & 25.16 \\
Standard & 1.58 & 1.58 & 1.58 & 1.58 & 1.58 & 1.58 \\
Error & & & & & & \\
Median & 24.40 & 24.40 & 24.40 & 24.40 & 24.40 & 24.40 \\
Standard & 5.47 & 5.47 & 5.47 & 5.47 & 5.47 & 5.47 \\
Deviation & & & & & & \\
Sample & 29.97 & 29.97 & 29.97 & 29.97 & 29.97 & 29.97 \\
Variance & & & & & & \\
Kurtosis & -0.86 & -0.86 & -0.86 & -0.86 & -0.86 & -0.86 \\
\hline
\end{tabular}




\begin{tabular}{|l|l|l|l|l|l|l|}
\hline Skewness & 0.44 & 0.44 & 0.44 & 0.44 & 0.44 & 0.44 \\
Range & 17.00 & 17.00 & 17.00 & 17.00 & 17.00 & 17.00 \\
Minimum & 18.00 & 18.00 & 18.00 & 18.00 & 18.00 & 18.00 \\
Maximum & 35.00 & 35.00 & 35.00 & 35.00 & 35.00 & 35.00 \\
Sum & 301.90 & 301.90 & 301.90 & 301.90 & 301.90 & 301.90 \\
Count & 12.00 & 12.00 & 12.00 & 12.00 & 12.00 & 12.00 \\
Confidence & 3.48 & 3.48 & 3.48 & 3.48 & 3.48 & 3.48 \\
Level (95.0\%) & & & & & & \\
\hline
\end{tabular}

Interestingly, it is observed that all the descriptive statistics of length of notes are same for different strings. This implies that for every string though the same notes are located at different lengths but the range of variation of the note length for all strings is in the similar range. This gets supported by the fact that every note has a certain frequency and these frequencies obey a certain logical structure (Lahdevaara 2014). Inspite of the statistical data being the same, the distribution of length of node for every string generates different music. On analyzing different pluck locations

\section{References}

Bhattacharya, K.: Sound, India: Hindustan Publishing Concern (1989).

Blatter, A.: Revisiting music theory: Basic Principles, 2nd Routledge (2016).

"Determining Pitch" (2017)., from http://ffden-2.phys.uaf.edu/webproj/212_ spring_2014/Claressa_Ullmayer/Mersenne\%27s\%20 Laws.html

Retrieved January 07, 2018

Drozdowski, T.: Don't Fret It! 10 Things You Should Know About Frets. From

http://www.gibson.com/News-Lifestyle/Features/enus/10-things-you-should-know-about-frets-0705-1. aspx

(2012). Retrieved January 07, 2018

Gualandri D. (2016). Analysis of an Acoustic Guitar. https://courses.physics.illinois.edu/phys406/sp2017/ Student_Projects/Spring13/Dan_Gualandri_P406_ Final_Project_Report_Sp13.pdf. Retrieved January 07, 2018

Gunther, L.: The Vibrating String. In The Physics of Music and Color, pp. 11-62. Springer Science+Business Media, LLC. From https://www.coursehero.com/ file/21192809/9781461405566-c1pdf/

(2012).

Retrieved January 07, 2018

Herman R. (2012). The Harmonics of Vibrating Strings: An Introduction to Mathematical Physics Via Oscillations. of the guitar string, Gualandri reported that the brighter notes is caused by a stronger presence of higher harmonics (Gualandri 2016). Combination of the various notes of music that generates different frequencies creating pitch variation leads to the holistic sound of guitar.

\section{Conclusion}

Music is an art form based on scientific and mathematical concepts. On analyzing the stringed and fretted musical instrument, guitar, various numerical aspects are revealed. A mathematical relation governing the variation of thickness of the strings exist. The placement of the frets are based on a specific mathematical pattern. The length variation of the musical notes on every guitar string also follows a rhythmic pattern. Tension and mass density of a string, harmonics and frequency of the various guitar strings are among the physical factors that are responsible for generating the mathematical pattern existing in the musical aspect of a fretted and stringed instrument.

http://people.uncw.edu/hermanr/phy311/ MathPhysBook/MathPhys_Main.pdf. Retrieved January 07, 2018

Hokin, S.: Waves and Sound: The Guitar. Retrieved from Samuel Hokin's website: http://www.bsharp.org/physics/guitar (2018).

Lahdevaara, J.: The Science of Electric Guitars and Guitar Electronics.

http://guitarscience.net/papers/guibook.pdf (2014). Retrieved January 07, 2018.

McNeil: The Physics of Guitar Strings. http://physics. csustan.edu/Roger/PhysicsofGuitarStrings.pdf (2017). Retrieved January 07, 2018.

Nave R.: Fret Rule for Guitars. http://hyperphysics.phy-astr. gsu.edu/hbase/Music/guita.html\#c3 (2017). Retrieved January 07, 2018.

Noyce. I.: String Tension and Tuning. http://www.noyceguitars.com/Technotes/Articles/ T3.html (2017). Retrieved January 07, 2018.

Savage, N. \& Andrew, C.: Fingers, Frets, And Strings. https://www.guitarlessons.com/guitar-lessons/ beginner-guitar-quick-start-series/fingers-frets-andstrings

(2017). Retrieved January 07, 2018

Varieschi, G. \& Gower, C.: Intonation and compensation of fretted string instruments. Am. J. Phys. 78 (1): 47-55 (2010). Retrieved from https://arxiv.org/ pdf/0906.0127.pdf 


\section{旬 \\ CHITKARA}

\section{Mathematical Journal of Interdisciplinary Sciences}

Chitkara University, Saraswati Kendra, SCO 160-161, Sector 9-C, Chandigarh, 160009, India

Volume 8, Issue 2

March 2020

ISSN 2278-9561

Copyright: [@ 2020 Anindita Roy Chowdhury and Naresh Sharma] This is an Open Access article published in Mathematical Journal of Interdisciplinary Sciences by Chitkara University Publications. It is published with a Creative Commons Attribution- CC-BY 4.0 International License. This license permits unrestricted use, distribution, and reproduction in any medium, provided the original author and source are credited. 